\title{
Rousseau et Diderot, deux positions de légitimation face à l'espace public
}

\author{
Anne Elisabeth Sejten (Roskilde University, Danemark)
}

\begin{abstract}
The quarrel between Rousseau and Diderot - these inseparable friends only to become enemies - constitutes a legendary topos of philosophical breaksups over-shadowed by wicked slander. If Rousseau's famous Letter to d'Alembert in 1758 traces his growing disagreement with the inner circle of encyclopaedists, further confrontations seem lost in mean accusations that discredit both sides. However, when digging a little deeper in the texts on both sides, it is not only possible to reconstruct different views about philosopher's morality, but also to relate to the complex nature that characterised the public sphere in eighteenth century France.

The purpose of this paper is exactly to examine the respective positions of Rousseau and Diderot as distorted voices in a society, in which the public sphere was actually more private than public. The first part of the study draws on the German philosopher Jürgen Habermas' seminal work on the transformation that affected the public sphere along European bourgeois countries in quite different ways during the eighteenth century. French salon culture and its essentially private spaces urged Rousseau and Diderot to invent original writing strategies in order to justify themselves. The second and major part of the paper, accordingly aims at analysing their literary choices, Rousseau in reinventing the autobiographical approach, Diderot by writing on the Roman stoic philosopher Seneca. Both philosophers are extremely aware of the need to protect their philosophical legacy and, in doing so, each is eventually playing the role of the contra-model of the other when facing the question of moral and intellectual integrity.
\end{abstract}

Keywords: public sphere, intellectuality, independency, morality, autobiography

\section{Introduction}

La relation entre Rousseau et Diderot se dérobe à l'étoffe mythologique des brouilles philosophiques, tant il existe d'anecdotes sur ces «frères ennemis ${ }^{1}$, inséparables amis qui finissent lamentablement par devenir des ennemis irréconciliables. Au départ tout respirait l'entente, lorsque peu après l'arrivée de Rousseau à Paris en 1742 les deux hommes se liaient d'une amitié de plus en plus profonde. Ils se voyaient tous les jours, se promenaient tout en causant à perte de vue, ils jouaient aux échecs au Café de la Régence - comme le fera le Neveu de Rameau - et lorsque Diderot devait aller en prison, condamné pour sa Lettre sur les

\footnotetext{
${ }^{1}$ L'expression est de Jean Fabre qui a retracé l'histoire des rapports des deux philosophes à partir de la rencontre au Café de la Régence en 1742 jusqu'à la rupture officielle en 1757 (voir Fabre 1961).
} 
Aveugles $^{2}$, Rousseau venait le voir, à pied, chaque jour. Autant de témoignages de l'âge d'or d'une amitié sans failles, telle que Diderot nous la résume ici : «c'était alors que j'étais lui, qu'il était moi [...] Nous n'avions qu'une bourse »(Diderot $2010: 852$ ).

L'élan passionné de Diderot se limite à la jeunesse - «l'amitié est la passion de la jeunesse » (ibid.) - car, incontestablement, cette affinité profonde qui les reliait allait se gâcher jusqu'à se consumer dans la rupture irréparable. Bien qu'il collaborât à l'Encyclopédie, Rousseau se distanciait en réalité de plus en plus des idées auxquelles se rayaient les encyclopédistes. Sa fameuse Lettre à d'Alembert marqua, en 1758, l'éclatement du désaccord avec lui et le cercle des encyclopédistes. Par opposition à d'Alembert, Rousseau mettait en garde, au sujet de l'établissement d'un théâtre à Genève, le danger lié au théâtre pour corrompre la moralité en société. Cette querelle fait soupçonner l'intérêt politique que Rousseau, très en avance sur son temps, portait pour les grands clivages sociaux dans le cadre monarchique. Derrière le critère quelque peu naïf, romantique et potentiellement conservateur de la vie naturelle, à laquelle un théâtre serait une menace, s'entrevoit une critique du caractère superficiel d'une société s'adonnant aux spectacles, de la prééminence des grandes villes sur les petites villes et, surtout, de la suprématie de l'aristocratie qui s'adonne aux loisirs sur le peuple qui travaille (Lepan 2015 : 109).

L'indignation sociale ne justifie cependant que partiellement la réaction violente de Rousseau vis-à-vis des encyclopédistes. D'autant plus que le travail rédactionnel et éditorial que représente 1'Encyclopédie constituait une société de gens de lettres, on se demande pourquoi ce milieu intellectuel fécond ne parvenait pas à encadrer un vrai débat sur les principes moraux que Rousseau défend dans sa Lettre à d'Alembert, voire à faciliter un échange d'idées continu et honnête avec Diderot. Pourquoi les nombreux rapports de force qui traversent l'Encyclopédie ne se limitaient-ils pas au pouvoir séculier et ecclésiastique au lieu de diviser les rédacteurs et collaborateurs entre eux ? Pourquoi fallait-il se séparer en ennemis au lieu d'engager une discussion sur les questions morales qui préoccupaient autant Diderot que Rousseau?

L'explication que nous allons poursuivre touche au caractère fort ambigu qui caractérisait l'espace public à l'époque des Lumières, dans la mesure où l'absence d'une séparation suffisamment consistante entre sphère publique et sphère privée semble influer non seulement sur les positions philosophiques défendues par les deux protagonistes, mais également sur leurs stratégies d'écriture respectives. Explication, certes, décalée et agissant comme à distance par rapport à la polémique entre les deux hommes de lettres, mais qui néanmoins reflète des conditions spécifiques pour se parler, pour discuter, voire pour se disputer. Force nous sera donc de nous interroger, dans les pages qui suivent, sur les rapports qui s'articulent entre l'écriture et l'espace public dans la malheureuse querelle entre Rousseau et Diderot tout en saisissant les positions philosophiques qui s'y profilent. À cette fin, nous allons revenir aux thèses de celui qui apparaît comme le penseur par excellence de la sphère publique : le philosophe allemand Jürgen Habermas.

\footnotetext{
${ }^{2}$ Lettre sur les aveugles à l'usage de ceux qui voient, publiée en 1749, fut immédiatement interdite pour blasphème, car s'il est vrai que le déisme des Pensées philosophiques avait sauvé Diderot à la persécution, ses propos sur les aveugles, ne donnent pas lieu à se méprendre, comme par exemple il fait dire à l'un de ses amis aveugles : «si voulez que je croie en Dieu, il faut me le faire toucher». Pour une lecture des rapports qu'entretient la critique religieuse dans ce texte de jeunesse avec une pensée esthétique, voir Sejten 1999.
} 


\title{
2. Où se parler ? Comment engager une vraie discussion ?
}

Ce qui déclenchait la rupture définitive de Rousseau avec Diderot se perd dans une myriade de malentendus et d'intrigues autour de la comtesse d'Houdetot, la première Sophie bien réelle de qui Rousseau s'était passionnément épris - histoire d'amour non-partagée et douloureuse pour Rousseau qui se sentait trahi par une indiscrétion de la part de Diderot ${ }^{3}$. Il ne s'agit certainement pas ici de retracer les multiples avatars de cette histoire de brouillerie, ni de scruter le fondement, vrai ou non, des propos ambigus, sinon mesquins, qui l'alimentent des deux côtés, si bien que celui qui critique l'autre finit par se voir discrédité par les mesquineries auxquelles il se livre. Ces épisodes cachent cependant mal un malaise plus profond, le manque de confiance qui avait déjà éloignés les deux amis l'un de l'autre. En tout cas, les invitations réitérées de la part de Diderot pour se voir demeuraient détournées par Rousseau.

Un premier signe, véritable symptôme, qui témoigne des difficultés pour se parler réellement, en hommes d'amis autant qu'en philosophes, se manifeste précisément dans les propos d'un Diderot soucieux, sinon désespéré, pour rentrer en contact avec un Rousseau qui persiste à garder son isolement. Lorsque Rousseau, déterminé à quitter Paris, laissait Diderot seul face aux difficultés qui entouraient la publication de plus en plus délicate des volumes en cours, celui-ci était en réalité plus affligé que fâché d'être abandonné à un moment où il avait besoin de son ami à ses côtés comme directeur de l'Encyclopédie. Il est vrai que l'on cite souvent la dernière phrase d'une lettre que Diderot avait écrite à Rousseau le 10 mai 1757 comme preuve de sa désapprobation sarcastique de l'isolement de celui qu'il considérait encore être son ami : "Adieu le citoyen. C'est pourtant un citoyen bien singulier qu'un ermite » (Diderot 1876 : 439). Or c'est peut-être d'aller un peu trop vite. Car plus révélatrices de sa frustration, les lettres de Diderot à cette période révèlent ses efforts répétés pour convaincre Rousseau de revenir sur sa décision. Il suffit de se rapporter à la même lettre dont est issue la maudite sentence citée ci-dessus pour s'assurer de l'extrême gentillesse avec laquelle Diderot essaye par tous les moyens de permettre à Rousseau de passer à Paris, ne serait-ce que pour quelques jours :

\begin{abstract}
Savez-vous ce que vous devriez faire ? Ce serait d'arriver ici et d'y demeurer deux jours incognito. J'irais samedi vous prendre à Saint-Denis, où nous dînerions et de là nous nous rendrions à Paris dans le fiacre qui m'aurait amené. Et ces deux jours, savez-vous à quoi nous les emploierions? À nous voir, ensuite à nous entretenir de votre ouvrage; nous discuterions les endroits que j'ai soulignés et auxquels vous n'entendrez rien si nous ne sommes pas vis-à-vis l'un de l'autre. (Ibid.)
\end{abstract}

Diderot avait manifestement besoin de son ami pour discuter dans l'intimité intellectuelle et amicale qui était devenue la leur, tout en ayant néanmoins le pressentiment que, sans ce tête-à-tête, les deux s'entendraient mal. Prophétie qui se verra vérifiée par la suite : Rousseau tient à l'isolement qu'il s'est imposé, et, intentionné ou non, Le fils naturel

\footnotetext{
${ }^{3}$ Cette affaire se consume par le rejet de Mme d'Épinay qui mit fin au séjour de Rousseau à l'Hermitage qu'elle avait mis à sa disposition en 1756. Lors de l'absence de leurs amants respectifs, Mme d'Épinay et Mme d'Houdetot se trouvaient en compagnie avec Rousseau, et bien que sa passion pour Mme d'Houdetot ne fût pas partagée et restât de nature platonique et amicale, Rousseau finit maladroitement par provoquer la colère de tous, non seulement celle des deux amants, qui furent le Baron Grimm et Saint-Lambert partis en guerre, mais donc aussi celle de Mme d'Épinay qui lui tourna le dos. Le rôle de Diderot fut aussi crucial qu'involontaire car relevant d'un malentendu : Diderot croyait à tort que Rousseau avait suivi son conseil de se confier à SaintLambert, et lorsqu'il en parla à ce dernier, l'erreur fut irréparable.
} 
de Diderot parvient par la suite à aggraver leur relation au moyen d'une seule réplique : «L'homme de bien est dans la société et il n'y a que le méchant qui soit seul » (Diderot 1757 : 97). Rousseau se sentait irréparablement visé par ces mots, et dès lors, sujet à des satires cruelles, il allait développer une attitude de plus en plus paranoïaque et afficher envers Diderot et les collaborateurs de l'Encyclopédie un ton exaspéré d'auto-défense.

Tout se passe comme si ces deux amis de jadis n'avaient pas eu accès à une sphère de civilité neutre, pour se parler, pour argumenter et disputer en bons philosophes. On dirait même que l'absence d'un espace public les aurait empêchés de s'entretenir en privé, comme l'aurait tant souhaité Diderot, car l'espace privé non plus n'était mis à l'abri des intrigues et des intérêts de pouvoir. Ce malaise qu'on ressent très fort des deux côtés renvoie effectivement au caractère encore très fragile qui distingue l'espace public en France à l'âge des Lumières. Dans son ouvrage sur l'espace public de la société bourgeoise, Jürgen Habermas qualifie justement la sphère publique du dix-huitième siècle français de "privée » - «private Offentlichkeit »- ce qui est évidemment une désignation paradoxale, puisque le propre d'une «Offentlichkeit » réside dans son caractère public, ouvert et autonome, mais il est certain que les difficultés d'une presse soumise à la censure nourrissait en France la culture des salons que l'on peut à juste titre voir comme une multiplicité d'espaces privés qui tant bien que mal font circuler des discours en cours, mais aussi quantité de rumeurs et d'histoires difficilement vérifiables (Habermas 1978 : § 9).

Derrière les attitudes opposées - Diderot plein d'ardeur pour faire retenir Rousseau à Paris, Rousseau s'obstinant dans son retrait - on devine ainsi des convictions tout aussi opposées quant aux perspectives pour mener à bonne fin toute discussion ayant un intérêt général. Leur divergence est peut-être due au fait qu'ils vivent et jugent différemment les conditions pour se parler. Que la séparation entre espace public et espace privé ne soit pas suffisamment assurée, voire garantie, devait nuire aux aspirations démocratiques d'un Rousseau, privé des droits qui lui aurait valu la sécurité de sa personne autant que la liberté de sa parole civique. D'esprit bien plus républicain, Diderot avait plus de confiance dans les effets de ses discours indépendamment des cercles sociaux dans lesquels ils étaient tenus. Il ne fuyait pas comme Rousseau ces nouveaux espaces de rassemblement intellectuels que représentent les Salons, croyant, comme le remarque à juste titre Habermas, dans leur fonction de divulgation pour faire circuler de nouvelles idées et pensées : "Nos écrits n'opérant que sur une certaine classe de citoyens, nos discours sur toutes » (Diderot cité par Habermas $1978: \S 5)$.

Bien que les attributions respectives de 'démocratique' et de 'républicain' ne soient applicables au plein sens des termes qu'après la Révolution, elles semblent préfigurer une tension au sein du désaccord entre les deux philosophes. Aussi, il est intéressant de rappeler que les critères de ce qui établit la sphère publique selon Habermas s'inspirent de la philosophie politique de Rousseau. La sphère publique renvoie justement à cet espace civique qui est fondé sur la séparation du public et du privé, qui jouit d'une liberté et d'une autonomie par rapport aux pouvoirs étatiques, qui est ouverte à tous, et qui, de ce fait, favorise une discussion en faveur du bien de la société (Habermas 1978 : § 4-7). Cette définition de la sphère publique embrasserait les raisons qui semblent s'inscrire au creux du refus de Rousseau pour socialiser. On dirait même que l'idée de la discussion libre et tenue à l'écart des intérêts particuliers s'articulerait, bien que négativement et de façon anticipée, dans les phobies de Rousseau. 
Si l'étouffement relatif d'une sphère publique n'a pas favorisé le dialogue sobre entre Diderot et Rousseau - bien au contraire, il l'aurait empoisonné - il n'en demeure pas vrai que ce même espace public, en quelque sorte court-circuitée par des publics semi-privés des salons, les aurait poussés à inventer des stratégies d'écriture fort originales pour se justifier, ne serait-ce qu'aux yeux de la postérité. C'est ainsi que Rousseau et Diderot, tous deux, se lancent dans des projets de légitimation bien particuliers, diamétralement opposés, l'un mobilisant l'écriture autobiographique, l'autre en se laissant glisser dans la figure du double à qui il se dérobe tout en s'y identifiant. Bien que les grands textes qui en découlent ne puissent se défaire des traits défavorables d'autojustification pénible, voire d'attaques indignes, ils ne tournent pas moins autour des questions sur le rôle du philosophe en société, sa moralité, et intégrité philosophique. Voilà donc que l'état encore rudimentaire d'une sphère publique et la discussion philosophique sur des questions morales s'articulent dans deux positions de légitimation antinomiques, celle d'une écriture du moi face à celle d'une écriture du double.

\section{Rousseau ou l'écriture du moi}

Le cours des choses nous invite à commencer par Rousseau, car c'est grâce à son projet autobiographique que les confrontations avec Diderot nous sont transmises. Fidèles au genre autobiographique, les Confessions, qui vont en constituer un moment constitutif, relatent les incidents et les embrouillements qui composent la malheureuse querelle entre les deux philosophes pour lui conférer ainsi le statut officiel que nous connaissons. Rousseau s'y étale longuement sur ladite affaire de Madame d'Houdetot ${ }^{4}$, sur les vifs échanges entre lui et Diderot dans le sillage du Fils Naturel, enfin sur d'innombrables épisodes, propos et rumeurs qui font de Diderot et son entourage, avec Grimm, d'Holbach et Voltaire en première ligne, un véritable rempart de machinerie contre lui. Sorte de flux psychanalytique de paroles peu conscientes de leur contenu refoulé, les Confessions témoignent bien davantage de la hantise de complot presque permanente dans laquelle vivait Rousseau qu'elles nous fournissent un document historique crédible.

Pourtant, il faut rappeler que Rousseau se trouvait dans une situation paradoxale entre la célébrité et l'exclusion. D'une part, il supplante la personnalité publique qu'il était devenu après avoir emporté les deux concours de l'Académie de Dijon par ces deux Discours qui font de lui une notoriété internationale ; d'autre part, partiellement à cause des idées nouvelles qu'il avait présentées dans ces mêmes ouvrages, il se voit contraint à une vie de plus en plus errante, fuyant les instances aussi bien politiques que religieuses. A ces persécutions bien réelles s'ajoutent donc celles plus imaginaires de ses anciens amis philosophes. À qui donc veut-il s'adresser ? Effectivement, Rousseau n'a de cesse de s'adresser à un autre public, à un public différent de celui de la haute société qui tient les salons littéraires. Ne serait-ce donc en attendant qu'un tel public se forme qu'il se tourne vers l'écriture autobiographique ? A ce sujet, il est intéressant de noter que Habermas considère Rousseau comme un des premiers à avoir pensé au rôle de l'opinion publique (Habermas 1978: § 12). Dans son premier Discours, celui sur les sciences et les arts, Rousseau avait précisément évoqué l'opinion publique qui selon Habermas doit servir de médiateur entre l'État et les besoins légitimes de la société. Dans cette perspective, l'écriture autobiographique que Rousseau va cultiver

\footnotetext{
${ }^{4}$ C'est dans le Livre neuvième (premières parties) que Rousseau retrace en détails l'histoire de sa brouille avec les belles-sœurs Mme d'Houdetot et Mme d'Épinay, ainsi que celle avec Diderot en intégrant à son récit des lettres entières recopiées de leur correspondance.
} 
semble remédier à l'éloignement d'une sphère publique accomplie, car autant se parler à soimême, s'il n'y pas lieu de s'entretenir avec des pairs dans un espace muni d'une autorité publique à côté de celle de la cour et de la noblesse. En tout cas, dès la sentence inaugurale des Confessions Rousseau insiste sur l'originalité de son «entreprise »: "Je forme une entreprise qui n'eut jamais d'exemple » (Rousseau 1959a : 33). Cette entreprise, précisément, consiste à conjuguer « vérité » à la parole du « moi », car l'homme qu'il veut «montrer » au monde «dans toute la vérité de la nature » n'est autre que lui - « cet homme ce sera moi »moi, aussitôt repris par la première phrase du paragraphe suivant : «Moi, seul. » (Ibid.) Ni le privé, ni le public lui assurent une plateforme de parole, les deux étant lamentablement mélangés et pénétrés par des intérêts particuliers.

Mais si les Confessions s'ouvrent ainsi sur le leitmotive du moi, seule garantie du dire vrai à défaut d'une sphère publique, le but en est en quelque sorte de s'apprêter à être jugé sur la vie menée. Solennellement Rousseau se prépare, au moyen de l'écriture du moi, à se présenter devant ses lecteurs futurs formant un juge à la fois universel et transhistorique : "Que la trompette du Jugement dernier sonne quand elle voudra, je viendrai, ce livre à la main, me présenter devant le souverain juge » (ibid.). Dès le départ se dessine ainsi une affinité entre d'une part la figure du paria dont Rousseau avait fini par embrasser bien des traits et d'autre part l'attitude introvertie d'un moi par rapport à cette entreprise de raconter sa vie. L'isolement de Rousseau, son retrait des milieux intellectuels ne trouve pas seulement dans l'écriture autobiographique une activité qui à la fois renforce et récompense sa marginalité ; Rousseau va jusqu'à faire de l'isolement et de l'indépendance qui en découle un argument éthique, enfin cette exigence morale d'une incorruptibilité intellectuelle qui traverse tous ses écrits autobiographiques ainsi que sa pensée en général.

Effectivement, aussi bien dans les Confessions que dans les Dialogues qui leur font suite, les règlements de compte que Rousseau mène en filigrane avec ses redoutables amis vont servir de pôle opposé pour se construire sa propre plateforme morale justifiant les choix qu'il a pris pour s'écarter du cercle des encyclopédistes et se distancier de leurs façons d'agir. Du coup, on s'aperçoit à quel point Rousseau projette le conflit qui n'éclate qu'en 1757 sur des incidents antérieurs qui n'ont que très peu à faire avec Diderot et les encyclopédistes. Dans l'étude magistrale qu'Yves Citton a consacrée à la querelle entre Rousseau et Diderot et sur laquelle nous nous appuierons par la suite - l'auteur fait remonter l'affaire à 1752 déjà, plus précisément à la suite de la représentation du Devin du village devant le roi. Grâce à la satisfaction du roi, Rousseau était en mesure de recevoir une pension royale, possibilité que pourtant il laissait tomber en évitant de se présenter au roi ${ }^{5}$, et c'était précisément cet acte manqué qui avait suscité la désapprobation de Diderot. Mais alors que Diderot, certes sur un ton vif, mais en toute amitié, avait évoqué la responsabilité dans laquelle Rousseau se trouvait pour prendre soin de sa femme à son charge ${ }^{6}$, Rousseau aussitôt justifiait l'affaire en termes de «maximes »: («[q]uoique je fusse touché par son zèle, je ne pus goûter ses maximes » (Rousseau 1959a: 464). Même avant de raconter l'échange avec Diderot, Rousseau a déjà mobilisé toute une charte morale basée sur ses «principes » à lui :

\footnotetext{
${ }^{5}$ Voir Trousson $1988: 292$.

${ }^{6}$ C'est grâce au récit de Rousseau lui-même que l'indignation de Diderot nous est peinte : «Il me dit que, si j'étais désintéressé pour mon compte, il ne m'était pas permis de l'être pour celui de Mme Le Vasseur et sa fille ; et que je leur devais de n'omettre aucun moyen possible et honnête de leur donner du pain » (Rousseau 1765$1770: 464)$
} 
Je perdais, il est vrai, la pension qui m'était offerte en quelque sorte : mais je m'exemptais aussi du joug qu'elle m'eût imposé. Adieu la vérité, la liberté, le courage. Comment oser désormais parler d'indépendance et de désintéressement ? Il ne fallait plus que flatter ou me taire, en recevant cette pension [...] Je crus donc en y renonçant prendre un parti très conséquent à mes principes. (463)

Rousseau retourne ici les reproches que Diderot lui avait adressés en évoquant des bien grands mots qui visent implicitement la façon compromettante dont les encyclopédistes, fort dépendants du pouvoir monarchique, doivent naviguer en société afin d'obtenir et maintenir le privilège royal de l'Encyclopédie. La question que pose Rousseau, en racontant comment il se dérobait à l'offre du roi, devient celle de la moralité et de l'intégrité du philosophe : comment les philosophes qui prétendent vouloir éclairer l'humanité peuvent-ils accepter de dépendre du pouvoir royal ? Ou bien, en termes plus généraux, comment assumet-on son devoir moral si on est payé par le pouvoir? Du même coup, l'idéal d'une sphère publique s'affirme comme manque, car comment discute-t-on si on en est privé ?

Dans sa remarquable étude, Yves Citton a dégagé les quatre revendications qui se profilent dans la solennelle déclaration citée ci-dessus - revendications reliées entre elles et que Citton ne tarde pas à mettre en perspective par les Dialogues qui prolongent l'écriture autobiographique des Confessions, dans la mesure où le scénario dialogique à plusieurs voix que les Dialogues introduisent ne fait que démasquer les attaques tournées contre les encyclopédistes. Nous résumons donc ici l'analyse fort révélatrice de Citton. La première revendication qu'il commente réfère au "désintéressement », voire le refus de Rousseau de confondre moralité et profit, ou bien, comme Rousseau jugeant Jean-Jacques dira dans les Dialogues en visant les encyclopédistes : "de ne pas plier comme eux sa morale à son profit" (Rousseau 1959b: 886). Deuxième revendication repérée par Citton est celle de «l'indépendance » dont se réclame Rousseau. Effectivement, en refusant la pension royale pour gagner sa vie, Rousseau ne veut dépendre de personne. Citton montre comment ce critère communique avec l'éloge du solitaire que Rousseau fait dans les Dialogues, car si « le plus sociable et le plus aimant des humains » choisit de vivre seul, éloigné du monde, c'est parce que la solitude garantie l'indépendance et l'authenticité. Par contre, ce qui le révolte chez les encyclopédistes, c'est précisément le manque d'indépendance à laquelle ils s'exposent. Moralement atteints, Diderot et ses amis Grimm et d'Holbach socialisent avec «le plus grand monde», mais au prix d'éternels compromis. Jean-Jacques, par contre, tel qu'il juge Rousseau dans les Dialogues, a préféré aux avantages qu'aurait donnés une pension la souffrance de l'isolation, mais donc isolation qui lui permet « de se suffire à soi-même » (Rousseau 1959b : 590 et 435). Ensuite, troisième revendication de la «vérité » se déduit en quelque sorte de celle de l'indépendance, puisque seule l'indépendance autoriserait de relier vie et vérité. De ne pas essayer de se retirer de la vie sociale et de tous les conflits qui la constituent signifierait servir le pouvoir au lieu servir la vérité. En tant qu' « étranger et vivant en France », et plus profondément en tant que socialement exilé, Rousseau déclare précisément dans les Confessions «[s]a position très favorable pour oser dire la vérité » (Rousseau 1959a: 492). Enfin, quatrième revendication se rapporte à la nécessité d'être «conséquent » à ses principes. En effet, si Rousseau réussit de donner l'image de lui-même comme quelqu'un moralement capable d'accomplir le parfait accord entre ce qu'il dit et ce qu'il fait, entre ses principes et ses actions, c'est qu'il dénonce les inconséquences qui cachent mal l'hypocrisie des philosophes autour de Diderot et consorts. (Voir Citton 2004 : 58-62) 
Voici donc comment cette écriture du moi, dans sa prétention de dire vrai sur « tel que je fus » accouche d'un véritable programme moral, lequel, à suivre l'interprétation d'Yves Citton, s'inscrit dans ce que Rousseau nomme lui-même sa «réforme », à savoir cette « grande maxime de morale » qui consiste à « éviter les situations qui mettent nos devoirs en opposition avec nos intérêts »(1959a: 91-92). Effectivement, si l'éthique de Rousseau est « essentiellement négative » (Citton 2004 : 59), étant donné qu'elle identifie ce qui doit être évité, plutôt que ce qui doit être accompli, elle se mesure en même temps négativement par rapport à l'idéal d'un débat public, libre et indépendant, constitutif de la démocratie délibérative, donc entièrement utopique dans le contexte du dix-huitième siècle français demeure utopique.

\section{Diderot ou une écriture du double}

L'entrée de Diderot dans l'affaire des brouilles naît en réaction à Rousseau. Sans l'écriture du moi initiée par Rousseau, il n'y aurait probablement pas eu celle du double par laquelle Diderot entend se défendre. Cela n'implique pas que Diderot se comporte beaucoup mieux que Rousseau dans la mesure où lui aussi va se livrer à calomnier un homme qui ne peut se justifier. Pourtant, là aussi, il faudra prendre en considération les conditions historiques qui caractérisent un espace public qui n'en est pas véritablement un. Ces deux grands écrivains semblent effectivement être très conscients de la fragilité qui menace leur renommée. Sans structures solides de la sphère publique, ils se trouvaient dans la nécessité de prendre soin de leur héritage philosophique. Diderot précise lui-même ce besoin de gérer sa réputation posthume : «Plus jaloux de préparer des regrets après ma mort que d'obtenir des éloges de mon vivant» (Diderot 2010 : 661). Il convient donc de préciser les circonstances spécifiques dans lesquelles Diderot allait réagir et donner libre cours à son indignation, à la fin de sa vie, contre Rousseau.

Rousseau venait de mourir en 1778, alors que le bruit de ses Confessions avait couru avant leur publication; il avait lui-même lu des passages dans des cercles privés, passages bourrés d'attaques malicieuses renouvelées contre Diderot et ses amis, si bien qu'on craignait des révélations. Quelle machinerie pouvait sortir de ces comptes que Rousseau réglerait avec le tout-Paris? Mme d'Épinay avait même réussi à faire interdire ces lectures par la police. Pourtant, après la mort de l'auteur, il n'y aurait personne à tenir responsable. C'est probablement la raison pour laquelle Diderot, bien qu'il eût décidé de se taire sur la brouille entre lui et Rousseau, prend la plume. Du coup, il se trouve dans une situation analogue à celle de Rousseau, car sans savoir à quel public il peut s'adresser. Et comme il s'était retiré à la campagne pour travailler en solitaire - «J'étais à la campagne, presque seul, libre de soins et d'inquiétudes » (Diderot 2010: 661), il se trouve paradoxalement là aussi dans un encadrement comparable à celle de l'isolement de Rousseau. Il y va de sa renommée, également mise en question.

Diderot sentait donc la nécessité de défendre les choix qu'il avait pris et de revendiquer, face à la postérité, son intégrité morale et philosophique que Rousseau ne cessait de persifler. Même s'il fait semblant de ne pas y attacher beaucoup d'importance (« ce n'est point par crainte d'être maltraité dans l'écrit posthume de Jean-Jacques qui m'a fait parler » (725)), sa réputation posthume lui importe : «Que Jean-Jacques dédaigne tant qu'il lui plaira le jugement de la postérité, mais qu'il ne suppose pas ce mépris dans les autres » (724). C'est ainsi que Diderot pour protéger son héritage va consacrer les dernières années de sa vie à 
écrire ses confessions à lui : "Ce n'est point une satire que j'écris, c'est mon apologie » (730). Sa démarche est cependant très différente de celle de Rousseau, façon signée Diderot, étant donné qu'il compose son autobiographie autour de la figure du philosophe romain Sénèque. Contre Rousseau, il se crée un double de Sénèque, ce qui permet un récit à deux voix, la sienne se réfléchissant et se comprenant à travers l'histoire tourmentée de Sénèque. $\mathrm{Au}$ fond, l'approche dialogique a toujours été la méthode philosophique de Diderot, Rousseau ayant probablement été l'un de ses premiers «alter ego », le double qui l'élucide sur luimême, et dans cette ultime confrontation avec Rousseau, Diderot se dédouble dans une œuvre qui est magnifiquement dialogique. L'écrit fut publié en 1779 sous le titre de 1'Essai sur la vie de Sénèque, dont une version révisée et élargie parut en 1782, deux ans avant la mort de Diderot, sous le titre de l'Essai sur les règnes de Claude et Néron, entièrement imprégné de la hantise de Rousseau.

Ce n'est guère un hasard que le personnage philosophique fort controversé sur qui Diderot charpente sa réfutation de Rousseau implique une inversion systématique des quatre revendications dégagées par Yves Citton qui ne manquera pas de nous le souligner: Qui pourrait réclamer d'être moins désintéressé que Sénèque ? Sénèque qui gagnait des fortunes en servant Néron le tyran? Qui pourrait réclamer d'être moins indépendant que Sénèque ? Assurément, il faut conclure avec Citton que la position du philosophe stoïque au sein de l'appareil d'État de Néron faisait de lui l'un de ses esclaves à la fois les plus puissants, les plus coupables, les plus infâmes qui étaient au service du despote. Et qui moins que Sénèque pourrait prétendre dire la vérité, occupé comme il l'était à étouffer les scandales meurtriers de la famille impériale ? D'abord, comme précepteur, il avait flatté le goût pervers de son élève, plus tard comme conseiller de l'empereur, il faisait tout ce qu'il pouvait pour dissimuler l'horreur et la terreur du règne de Néron. Alors, comment Sénèque, philosophe stoïque dans ses livres, le laquais du tyran par ses actions, pouvait-il réclamer la conformité entre ses discours et son comportement? (Voir Citton $2004: 71$ )

Effectivement, la tâche que Diderot s'était donnée en faisant l'éloge de Sénèque pas facile et d'autant plus catastrophique qu'il intentait de projeter sa propre vie sur celle de Sénèque afin de reprendre sa querelle avec Rousseau. Cette stratégie, cependant, souscrirait à ce que Citton nomme une «écriture du risque » (ibid.). Du coup, il s'avère moins intéressant de voir si Diderot réussit à justifier Sénèque que de dégager l'argumentation hautement ambivalente, illogique, presque contradictoire sur laquelle il centre son éloge, lorsqu'il est question des rapports entre les principes que donne le philosophe et sa conduite. Citton montre précisément que Diderot, se contredisant lui-même, en est parfaitement conscient, mais que les actions blâmables n'affectent pas forcément la justesse de ses propres principes. La pensée de Sénèque vaut par elle-même indépendamment de ce qu'il a été comme homme :

Que nous importe la contradiction vraie ou fausse de la conduite de Sénèque avec sa morale ? [...] Nous n'avons pas besoin de l'exemple de Sénèque pour savoir qu'il est plus aisé de donner un bon conseil que de le suivre. Tâchons donc d'en user à son égard comme avec tous les autres précepteurs du genre humain : faisons ce qu'ils nous disent sans trop nous soucier de ce qu'ils font. (Diderot 2010 : 780-781)

L'argument qu'avance Diderot ici met implicitement en cause l'exigence d'être conséquent à ses principes par ses actes, critère dont Rousseau se vantait être le gardien. C'est que les principes valent par eux-mêmes : les préceptes auraient leur propre rigueur en dépit des actions et de la faiblesse du précepteur. Aussi Diderot manquera-t-il pas de faire observer les inconsistances flagrantes de Rousseau lui-même, «ayant vécu vingt années avec des 
philosophes avant de devenir « antiphilosophe », «catholique parmi les protestants, protestant parmi les catholiques », "contre les spectacles, après avoir fait des comédies » (Diderot $2010: 728$ ) etc. Effectivement, admettre l'inconsistance entre principes et actions permet à Diderot d'axer autrement le critère de droiture morale : "Sénèque écrivait-il de belles et sages pages ? Quel était l'effet de ses textes sur leurs lecteurs ?» (Citton 2004: 72) Au lieu de réclamer le fait que les actions soient en accord avec les discours, Diderot discerne les effets qu'avaient les actions de Sénèque dans des contextes fort distincts. Contre la passion que Rousseau a pour l'unité et la cohérence, Diderot procède en examinant les fonctions liées aux rôles multiples qu'assumait Sénèque, comme moraliste, comme mari, comme précepteur, comme écrivain, comme ami, comme homme d'état afin de considérer leurs effets respectifs indépendamment de la façon dont toutes ces fonctions coexistent dans la même personne. Cette approche, ambiguë et potentiellement dangereuse, rejoint ce que Citton à juste titre résume sous les traits de « la politique du moins pire » (Citton $2004: 73$ )

Aussi Diderot transforme-t-il la revendication de vérité, capitale pour Rousseau, en une pragmatique d'effets. L'important consiste à minimiser le mal autant que possible. Diderot va jusqu'à jouer le rôle de l'avocat d'un homme qui reste la main droite du tyran malgré les crimes commis par son régime d'horreur, parce qu'il était « utile» :

Il était utile d'y rester pour l'Empire, pour la famille de Sénèque, pour ses amis, pour nombre de bons citoyens. [...] Démissionner de son poste reviendrait à faire] que la vertu demeure sans protecteur et que la scélératesse s'exerce sans obstacle [...]

Par son refus et par sa mort, Sénèque aurait été l'assassin de tous ceux qu'il eût abandonné à la férocité de Néron. Quelles auraient été les premières victimes d'une résistance inconsidérée ? Sa femme peut-être, ses frères, ses amis, une foule d'honnêtes et braves citoyens ». (Diderot $2010: 984$ )

Contrairement à ce que défend Rousseau, l'exigence éthique n'est pas une question de pureté ou d'innocence, mais constitue la stratégie du moins-pire, évoquée par Citton, stratégie selon laquelle il faut tout simplement éviter le pire. C'est pourquoi l'un des modes de défense que Diderot emploie fréquemment consiste à adopter le point de vue de Sénèque, à s'identifier avec lui en se mettant « à sa place»: «Mettez-vous à la place du philosophe, de l'instituteur et du ministre, et tâchez de conduire mieux que lui. » Car « [s]e mettre à sa place avant que de prononcer [est une] question sans laquelle on sera souvent injuste, [...] et l'on jugera les autres comme on ne voudrait pas être jugé. " D'où l'hypocrisie que Diderot dénonce chez ceux qui jugent Sénèque et ses semblables sans connaître les circonstances :

[c]e qui me confond, c'est la légèreté avec laquelle des hommes frivoles prescrivent des règles de conduite à des hommes d'une prudence consommée, et placés dans la plus orageuse des cours; et cela, sans en connaître les intrigues secrètes, les brigues, les mouvements, les caractères, les vues, les intérêts, les craintes, les espérances, les projets qui changent avec les circonstances, les circonstances qui changent d'un jour à l'autre; sans que leurs fausses conjectures sur ce qui se passe à deux lieues des bords de la Seine leur inspire la moindre incertitude sur ce qui s'est passé, il y a deux mille ans, sur les rives du Tibre. (Diderot 2010 : 707)

Cette argumentation, bien sûr, se soustrait à la possibilité même de juger Sénèque, parce que personne d'autre que lui aurait su ce qui était en jeu. Mais c'est peut-être le message qu'aurait désiré transmettre Diderot à Rousseau. Diderot ressentit fortement 
l'injustice d'un Rousseau qui le jugeait sans se mettre à sa place, notamment en ce qui concerne les négociations fort délicates qu'il dut entreprendre pour assurer la publication des tomes de l'Encyclopédie. Derrière la figure compromettante de Sénèque, Diderot s'identifie à toutes ces «sortes de courage » qu'il admire en lui - «celui des principes, celui du caractère, et celui du devoir » (Diderot 2010 : 753) - si bien qu'il finit par proposer Sénèque comme le grand modèle de sa pensée morale.

\section{Comment raconter sa vie ?}

Le caractère de l'essai de Diderot, son style d'imitation - comme s'il était Sénèque contraste fortement avec celui de l'élan solitaire chez Rousseau, soucieux de ne parler qu'à partir de son âme mise à nu par l'introspection. Autant conclure que la divergence entre les deux frères ennemis est aussi littéraire que moral. En s'attaquant à la question de l'intégrité morale, ils se confrontent à la question de savoir comment relater ce qu'on a été. Et tandis que les Confessions de Rousseau en général reposent sur l'idée d'une nature profonde inhérente à un individu pris dans son parcours chronologique, les confessions fragmentées de Diderot se calquent sur la personnalité de Sénèque qui agit constamment en fonctions des relations changeantes avec d'autres personnes.

$\mathrm{Au}$ lieu de se poser comme un cas unique et exemplaire, comme le fait incontestablement Rousseau, Diderot s'inscrit dans une durée et dans une tradition philosophique qui va de Sénèque à Montaigne et de Montaigne à lui-même (Delon 2010 : 1325). Cette durée, Diderot la thématise en voyant en Sénèque « un des plus doux liens entre mes amis et moi, entre les hommes instruits, de tous les âges » ou bien «l'organe de la justice des siècles » (Diderot 2010 : 664). Lorsque Diderot imite Sénèque il s'intéresse en réalité à des situations qui se répètent au cours des siècles, à échanger leurs places («si j'avais été à ta place et toi à la mienne » (ibid.)). Alors que Rousseau établit une sorte de tête-à-tête avec luimême pour se justifier, Diderot demeure ainsi fidèle à une sorte d'altruisme qui ne lui permet jamais d'être seul. Diderot s'inscrit dans une histoire, il imite les philosophes qui lui ressemblent. Il leur est fidèle, alors que Rousseau est fidèle à lui-même, mais tous deux restent fidèles à leurs convictions à partir desquelles ils s'affirment comme des êtres moraux. Définitivement, aussi bien par la forme littéraire que sur le fond des idées, les deux frères ennemis jouent les contre-modèles l'un de l'autre en abordant les questions de l'engagement moral. Ce qui est n'est pas le moindre accomplissement de l'héritage partagé au sein de leurs désaccords.

Il n'en demeure pas moins vrai que tous deux sont victimes de cet espace public qui leur fait défaut et les empêche de se croiser, de se parler et de se comprendre, car si Diderot ironise contre Rousseau qu' « [o]n ne s'améliore pas dans les bois » (Diderot 2010 : 727), lui aussi tâtonne dans le crépuscule ne sachant pas très bien sur quels lecteurs il allait pouvoir compter. Diderot insiste sur la nécessité de philosopher et d'agir en société, mais il se montre assez peu critique vis-à-vis des intérêts et pouvoirs qui porteraient atteinte au bon fonctionnement de l'espace publique, alors que Rousseau s'isole en raison des visions bien plus démocratiques. D'où également l'actualité de leur divergence. Tout comme Jürgen Habermas a évoqué, au sujet des sociétés actuelles, le danger d'une « refeudalisation » à cause d'un espace public de plus en plus perverti par des intérêts privés, la querelle entre Rousseau et Diderot rappelle que la sphère publique constitue une sorte d'idéal, qu'elle doit être acquise 
et continuellement défendue, y compris l'idée utopique d'un débat uniquement commandé par l'usage public de la raison, et non des intérêts particuliers.

\section{Bibliographie}

Citton, Yves. 2004. «Retour sur la misérable querelle Rousseau-Diderot : position, conséquence, spectacle et sphère publique. » Recherches sur Diderot et sur l'Encyclopédie, Paris : Société Diderot, vol 36.

Delon, Michel. 2010. « Notice», Euvres philosophiques (éd. Michel Delon), Paris : Éditions Gallimard (Bibliothèque de la Pléiade), p. 1314-1325.

Diderot, Denis. 1876. Correspondance générale, Euvres complètes (éd. Assézat et Tourneux), tome 19, Paris : Garnier frères.

Diderot, Diderot. 1757. Le fils naturel, Amsterdam : s.n.

Diderot, Diderot. 2010 [1782]. Essai sur les règnes de Claude et de Néron, Euvres philosophiques (éd. Michel Delon), Paris : Éditions Gallimard (Bibliothèque de la Pléiade), p. 658-1006.

Fabre, Jean. 1961. «Deux frères ennemis : Diderot et Jean-Jacques, Diderot Studies, vol. 3, Genève : Librairie Droz, p. 155-213.

Habermas, Jürgen. 1978. L'Espace public : archéologique de la publicité comme dimension constitutive de la société bourgeoise, traduction de Marc de Launay, Paris : Payot.

Lepan, Géraldin. 2015. Rousseau, une politique de vérité, Paris : Belin.

Rousseau, Jean-Jacques. 1959a [1782 et 1789]. Les Confessions (éd. Bernard Gagnebien et Marcel Raymond), Paris : Éditions Gallimard, coll. Folio (édition dérivée de la Bibliothèque de la Pléiade).

Rousseau, Jean-Jacques. 1959b [1782]. Rousseau, jugé de Jean-Jacques. Dialogues, Euvres Complètes, t. I, Paris : Gallimard (Pléiade), p. 657-992.

Sejten, Anne Elisabeth. 1999. Diderot ou le défi esthétique. Les écrits de jeunesse 1746-1751, Paris : Vrin.

Trousson, Raymond. 1988. Jean-Jacques Rousseau, Paris : Tallandier. 